\title{
Mejora de procesos de producción a través de la gestión de riesgos y herramientas estadísticas
}

\author{
Improving the processes of production through risk \\ management and statistical tools
}

\author{
Alexander D. Pulido-Rojano ${ }^{1 *} \quad$ Alex Ruiz-Lázaro $^{1} \quad$ Luis Eduardo Ortiz-Ospino $^{1}$ \\ Recibido 22 de septiembre de 2017, aceptado 8 de octubre de 2018 \\ Received: September 22, 2017 Accepted: October 08, 2018
}

\begin{abstract}
RESUMEN
Los enfoques de prevención de riesgos en actividades, funciones o procesos se han convertido en piezas fundamentales a la hora de minimizar la ocurrencia de eventos que son perjudiciales para las compañías. Cada producto no conforme está estrechamente ligado con eventos no deseados relacionados con uno o algunos de los factores que intervienen en el proceso. La identificación, análisis, evaluación, tratamiento, comunicación y monitoreo de estos eventos no deseados garantizarán el incremento de la calidad en los productos y la productividad en el proceso productivo. En este artículo, proponemos un diseño metodológico para la prevención de riesgos en procesos productivos. La metodología propone una forma novedosa de combinar el uso de herramientas estadísticas de calidad y la norma ISO 31000 de gestión de riesgos. La validación fue hecha sobre un proceso de envasado de productos lácteos. Las conclusiones de esta investigación muestran que el diseño metodológico propuesto es suficientemente flexible para ser adaptado a cualquier tipo de proceso de fabricación que se desea monitorear y mejorar.
\end{abstract}

Palabras clave: Control de calidad, proceso de gestión de riesgos, mejora de calidad, herramientas de estadísticas.

\begin{abstract}
Risk prevention approaches on activities, functions or processes are fundamental elements to prevent events that are harmful to companies. Each non-conforming product is related to undesirable events associated with the factors intervened in the process. The identification, analysis, evaluation, treatment, communication and monitoring of these unwanted events will help to increase the quality of the products and productivity in the production process. In this paper, we proposed a methodological design for the prevention of risks in production processes. The methodology proposes a novel way of combining statistical quality tools and the ISO 31000 standard for risk management. The validation was performed on a process of packaging of dairy products. The conclusions of this research show that the proposed methodological design is sufficiently flexible to be adapted to any type of manufacturing process that is desired to be monitored and improved.
\end{abstract}

Keywords: Quality control, risk management process, quality improvement, analysis tools.

\footnotetext{
1 Programa de Ingeniería Industrial. Universidad Simón Bolívar. Carrera 59 N 59-65. Barranquilla, Colombia. E-mail: apulido3@unisimonbolivar.edu.co; aruiz25@unisimonbolivar.edu.co; lortiz27@unisimonbolivar.edu.co

* Corresponding Author: apulido3@unisimonbolivar.edu.co
} 


\section{INTRODUCCIÓN}

La competitividad es actualmente el gran reto que enfrentan las organizaciones. La mejora continua en los procesos y productos se constituye como la clave para alcanzarla. Dentro de esta mejora continua, la calidad es sin duda pieza fundamental para satisfacer las expectativas de los clientes, de ahí que a través del tiempo las compañías busquen mecanismos para garantizarla y evitar productos defectuosos que se traduzcan en cuantiosas pérdidas, no solo a nivel financiero sino de imagen frente a los clientes [1-4].

La gestión de riesgos es una de las mejores prácticas que actualmente se llevan a cabo en todo tipo de organizaciones a lo largo y ancho del mundo. Su finalidad es que las organizaciones gestionen los riesgos tanto en su ambiente exterior o interior, con el fin de que se mitiguen aquellos eventos que puedan impactar negativamente el logro de sus objetivos $\mathrm{y} / \mathrm{o}$ que potencialicen aquellos eventos que puedan impactar positivamente el logro de los mismos [5, 6]. La gestión de riesgos ha sido ampliamente utilizada en el campo de las finanzas [7, 8], la gerencia de proyectos [9-11], la salud y la seguridad [12] y el sector de tecnología de la información [13]. La normativa internacionalmente aplicada en este sentido es la ISO 31000. Esta norma provee una guía para el establecimiento e implementación del proceso de gestión de riesgos, involucrando el establecimiento del contexto y la identificación, el análisis, la evaluación, el tratamiento, la comunicación y el monitoreo de los riesgos. Todo lo anterior enmarcado en un proceso sistemático y cíclico dentro de la organización, tal como se observa en Figura 1. La norma ISO 31000 toma en cuenta que todas las actividades de una organización implican riesgo, por lo tanto, describe cada componente del enfoque general del proceso de gestión de riesgos de la siguiente forma:

- Establecer el contexto. Establecer el contexto interno y externo de la gestión del riesgo en el cual tendrá lugar el resto del proceso. En esta parte del sistema se definen los criterios frente a los cuales se evaluará el riesgo y se define una estructura de análisis.

- Identificar riesgos. Se identifica con precisión dónde, cuándo, por qué, y cómo podrían los eventos que afecten a la organización prevenir, degradar, retardar o potenciar el logro de los objetivos organizacionales.

- Analizar riesgos. Determinar los controles existentes y analizar riesgos en términos de consecuencias y probabilidades en el contexto de esos controles. El análisis debería considerar el rango de consecuencias potenciales y cuán probable es que ocurran esas consecuencias.

- Evaluar riesgos. Comparar niveles estimados de riesgos contra los criterios preestablecidos.

- Tratar los riesgos. Aceptar y monitorear los riesgos de baja prioridad. Para otros riesgos, desarrollar e implementar un plan de administración específico que incluya consideraciones de fondo, reduciendo pérdidas potenciales.

Fuente: [5]

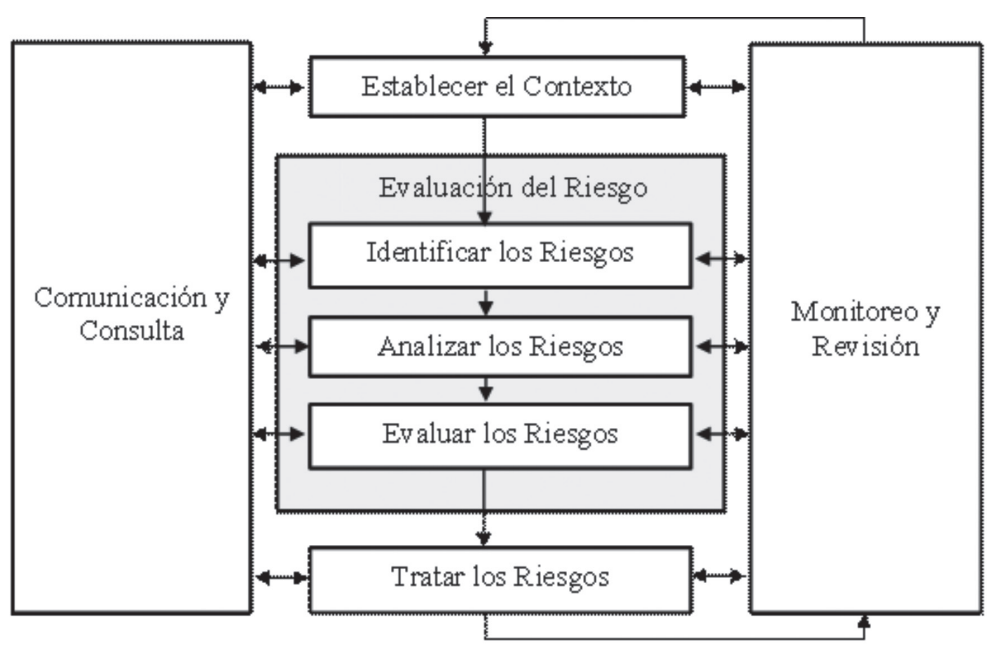

Figura 1. Enfoque general del proceso de gestión riesgos. 
- Comunicación y consulta. Comunicar y consultar con interesados internos y externos según corresponda en cada etapa del proceso de administración de riesgos y todos aquellos relacionados al proceso como un todo.

- Monitoreo y revisión. Es necesario monitorear la implementación de las etapas del proceso de gestión del riesgo. Esto es importante para la mejora continua.

Por otro parte, las herramientas estadísticas y de calidad han sido ampliamente utilizadas para apoyar las operaciones de los sistemas de producción y el cumplimiento de los requisitos de especificación de productos y servicios [14-21]. Estas herramientas permiten recopilar información y brindar bases para el control de calidad en los procesos. Las comúnmente adoptadas en las actividades de mejora de la calidad y utilizadas como soporte para el análisis y solución de problemas operativos en los más distintos contextos de una organización, son: La hoja de control (Hoja de recogida de datos), el histograma, el diagrama de Pareto, el diagrama de causa-efecto, el análisis por Estratificación, el diagrama de scadter (Diagrama de dispersión) y los gráficos de control [3, 4, 20-22].

Como hemos mencionado, la gestión de riesgo y las herramientas estadísticas han sido ampliamente utilizadas en muchos contextos distintos, sin embargo, es poco lo que se conoce acerca de su uso combinado para la mejora de procesos de producción que requieran la transformación y/o manipulación de las materias primas. En este sentido, el desafío surge cuando es necesario combinar técnicas estadísticas con normas de gestión para el cumplimiento de requerimientos de calidad y mejora en contextos nacionales e internacionales. Este trabajo presenta una metodología para la gestión de riesgos en procesos de producción. Esta metodología permite la integración de herramientas estadísticas y de calidad con el despliegue del proceso de gestión de riesgos contenido en la normativa ISO 31000. El enfoque propuesto es una herramienta útil para la detección y mitigación de producto no conforme, la identificación de posibles fuentes de no conformidades y la armonización de las filosofías de trabajo con requerimientos normativos. Para comprobar su efectividad, la metodología ha sido implementada en un proceso productivo, más precisamente, en una empresa del sector industrial de Barranquilla (Colombia) dedicada a la producción de productos lácteos.

Este artículo está organizado de la siguiente manera. La sección 2 describe la metodología propuesta para la mitigación de riesgos. La descripción del proceso de producción objeto de implementación se presenta en la Sección 3. Los resultados y el análisis son mostrados en la sección 4. Finalmente, la sección 5 resume las conclusiones de la presente investigación.

\section{DISEÑO METODOLÓGICO}

La metodología propuesta ha sido establecida como un proceso sencillo que resume las etapas a seguir para su implementación en cualquier proceso de producción. El enfoque de mejora inicia con la identificación y descripción de los productos y procesos a ser monitoreados, culminando con la revisión y el monitoreo de las acciones propuestas para mitigar los riesgos y las no conformidades identificadas en el proceso productivo (ver Figura 2). A continuación se describen cada una de las etapas:

- Identificación y descripción: En este primer paso se identifican los procesos y productos que serán monitoreados, se presenta una descripción detallada de las etapas de producción, actividades, métodos de trabajo y recursos utilizados. Identificando posibles fallas o desviaciones, riesgos y causas de no conformidades.

- Implementación y clasificación: Mediante la implementación de las herramientas estadísticas y de calidad, se clasifican las causas de las no conformidades o riesgos que fueron

Fuente: Autores.

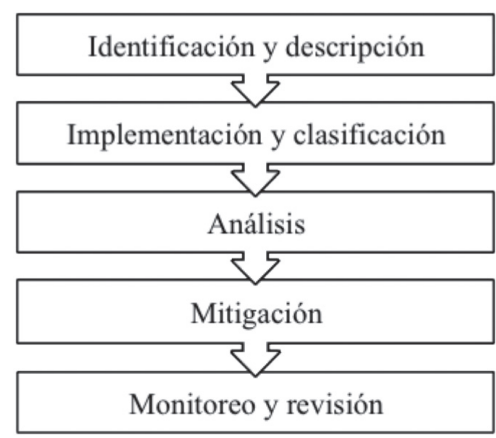

Figura 2. Pasos para la implementación del diseño metodológico. 
identificados en cada proceso seleccionado para ser monitoreado. Adicionalmente, se implementan instrumentos de monitoreo y control estadístico de procesos y se cuantifican las ocurrencias de las no conformidades. Las herramientas implementadas en esta etapa dependerán del tipo de proceso que se está analizando y de las necesidades del analista del proceso monitoreado.

- Análisis: En esta etapa se realiza un análisis estadístico de los procesos y un análisis de datos de la ocurrencia de los riesgos y las no conformidades. Lo anterior, con base en los resultados obtenidos en la etapa de implementación y clasificación.

- Mitigación: Con el fin de reducir los riesgos, desviaciones y no conformidades detectadas en el proceso productivo. Se propone el despliegue del proceso de gestión de riesgos de acuerdo con la norma ISO 31000 (ver Figura 1). La información de entrada en esta etapa corresponde a las salidas de las tres primeras etapas del diseño metodológico propuesto (Identificación y descripción, Implementación y clasificación, y Análisis). Asimismo, se establecen e implementan las acciones de contención, acciones de reacción y acciones de prevención para mitigar la ocurrencia de los riesgos.

- Monitoreo y revisión: Al ser un proceso de mejora continua, se propone realizar seguimiento y revisión de la eficacia de las medidas o acciones propuestas en la etapa de "Mitigación". Se recomiendan nuevas acciones en caso de que las acciones sugeridas en primer lugar no hayan sido efectivas, o si se desea actualizar el sistema en el paso de identificar nuevos riesgos.

Un esquema visual del enfoque metodológico propuesto en este trabajo se muestra en la Figura 3. Observe como en la etapa de "Mitigación" se implementa el enfoque de gestión de riesgos. Esta etapa toma como entrada toda la información de los procesos ya identificados como prioritarios; además, utiliza la información y el análisis obtenido de la implementación de las herramientas estadísticas.

\section{VALIDACIÓN DEL DISEÑO METODOLÓGICO}

La validación del diseño metodológico descrito en la sección anterior, se llevó cabo en una empresa industrial ubicada en la ciudad de Barranquilla, Colombia (no damos su nombre por razones de seguridad y efectos legales). Esta compañía está dedicada a la producción y comercialización de leche y sus derivados.

Desde las diferentes líneas de producción con que cuenta la empresa, la validación se centró en el estudio de la línea de producción de yogurt purepack, la cual está compuesta por cinco subprocesos: mezcla de yogurt, homogenización, pasteurización, maduración y envasado. El flujograma de producción se muestra en la Figura 4.

Fuente: Adaptado de [5, 22].

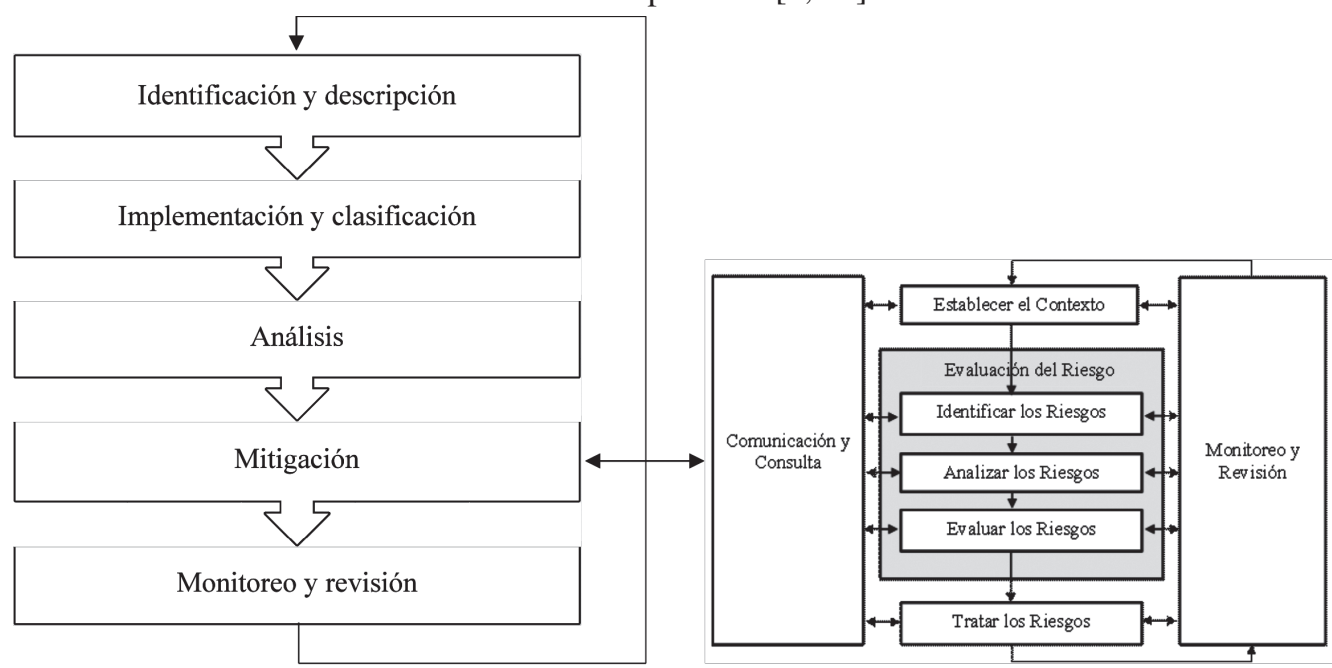

Figura 3. Enfoque del diseño metodológico. 
Fuente: Los autores.

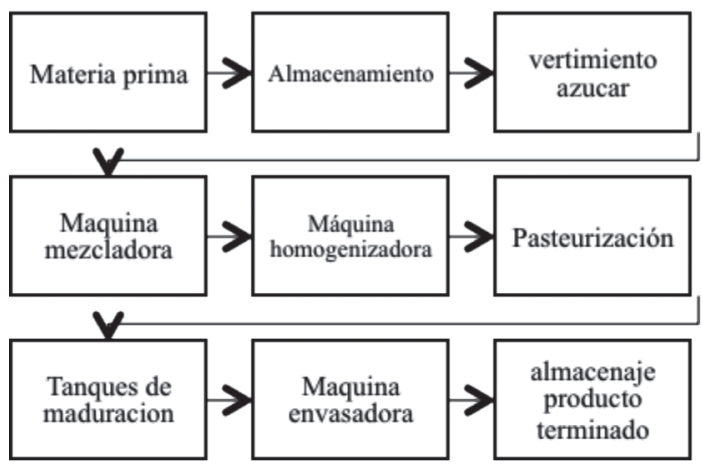

Figura 4. Flujograma línea de producción de yogurt purepack.

El proceso producción de yogurt purepack inicia con el bombeo semiautomático de leche hacia un tanque de almacenamiento. Luego, la leche es transferida por medio de tubos a la zona de preparación de yogurt, donde es introducida en el tanque de mezcla. Aquí la leche pasa a ser mezclada con azúcar. Posteriormente, la leche es transferida a la máquina homogeneizadora donde se balancea la leche entera con la leche con azúcar, una vez terminado este proceso pasa a la pasteurización.

Luego de ser pasteurizada, la leche mezclada se traslada a los buques maduradores, donde se cultiva el yogurt, se hacen variaciones de temperaturas para eliminar bacterias, y para hacer que el yogurt alcance y logre su maduración. Luego de estar listo el yogurt, éste pasa a la zona de envasado, donde es extraído por la máquina envasadora. Ésta, a su vez, extrae el yogurt mientras el operario principal carga la máquina con las cajas tetra pack. Se acciona el botón de encendido permitiendo que el mandril se ponga en marcha. Es necesario dejar pasar un intervalo de tiempo para que la resistencia de la máquina se caliente lo suficiente para que las actividades de sellado y fechado se realicen satisfactoriamente. Por último, las cajas siguen su curso por medio de una correa automática hasta llegar a la zona de recolección; donde operarios las inspeccionan y las ubican en las canastas de almacenamiento. Una vez apiladas, las canastas pasan a ser transportadas a la bodega de almacenamiento de producto terminado.

La validación de la metodología fue realizada sobre la línea de producción de yogurt purepack. Este proceso es crítico para la compañía debido al número de productos no conformes generados (ver más adelante en la subsección "Implementación y clasificación”).

\section{RESULTADOS Y ANÁLISIS}

El despliegue de cada etapa del diseño metodológico es presentado a continuación. Su aplicación es hecha en el subproceso de envasado de yogurt en cajas tetra pack.

\section{Identificación y descripción}

En esta primera etapa se identificaron las operaciones y herramientas de trabajo utilizadas en el subproceso de envasado, así como, los aspectos particulares y relevantes en cuanto al manejo y funcionamiento de la máquina de envasado. Además, se identificaron las etapas secuenciales del subproceso. También, se reconocieron las causas de no conformidades relacionadas con las condiciones de operación y el tipo de no conformidad causada por cada tipo de riesgo.

Los defectos comúnmente encontrados en el producto fueron: Mal sellado de boca en caja tetra pack, Fechado defectuoso, Rayado sobre la caja tetra pack por mala manipulación y Filtrado en la caja tetra pack. La descripción de cómo podrían presentarse estos defectos se muestra en la Tabla 1.

Como hemos mencionado en la sección "DISEÑO METODOLÓGICO”, toda la información recolectada en esta fase es utilizada como información de entrada para el desarrollo de las siguientes etapas.

\section{Implementación y clasificación}

En esta fase, se implementaron un conjunto de herramientas estadísticas y de calidad con el propósito de clasificar y analizar el subproceso. El diagrama de causa-efecto permitió apreciar con claridad las relaciones entre los riesgos o causas y las no conformidades identificadas en la fase de "Identificación y descripción". La Figura 5 y la Tabla 1 muestra un resumen en la clasificación de los riesgos y/o causas no conformidades tipificándolas en el enfoque de las 6 Ms.

El diagrama de Pareto permitió jerarquizar la ocurrencia de los defectos en el proceso de envasado (Mal sellado de boca en caja tetra pack, Fechado defectuoso, Rayado sobre la caja tetra pack y 
Fuente: Adaptado de [4].

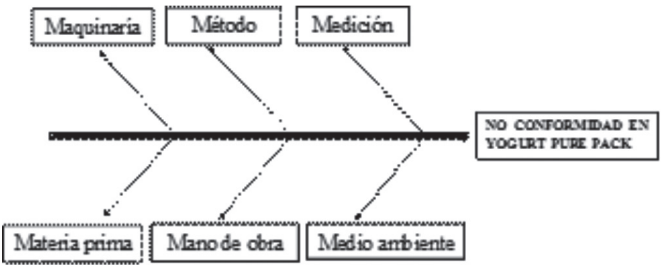

Figure 5. Diagrama causa-efecto para el proceso de envasado de yogurt Purepack.

Filtrado). Con la implementación de este diagrama se logró priorizar los defectos críticos en la línea de producción. La idea es concentrar la atención en aquellos defectos con una mayor ocurrencia y prioridad. El diagrama de Pareto se muestra en la Figura 6.

Asimismo, teniendo en cuenta las características del producto y proceso, se empleó el gráfico de control $n p$ para el monitoreo del subproceso de envasado de
Fuente: Los autores.

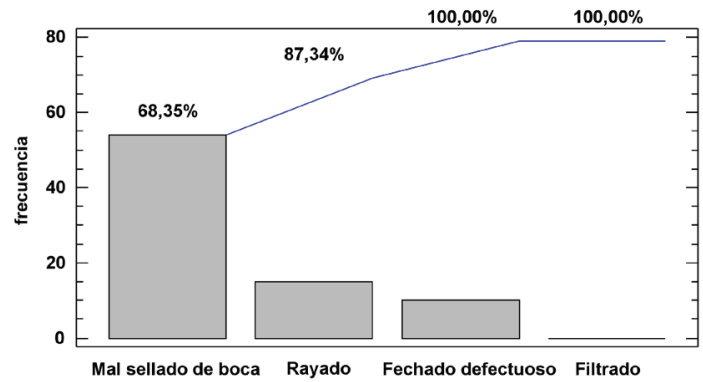

Figure 6. Diagrama de Pareto para defectos en el subproceso de envasado de yogurt purepack.

yogurt pure pack. El gráfico $n p$ evalúa el número de artículos defectuosos o en nuestro caso, el número de cajas de yogurt no conformes. Los subgrupos recolectados para el cálculo de los parámetros del proceso y los límites de especificación superior, inferior y central de la carta $n p$ se muestran en la Tabla 2 y las ecuaciones (1)-(4), respectivamente.

Tabla 1. Causas de no conformidades en el proceso de envasado de yogurt purepack.

\begin{tabular}{|l|ll|}
\hline \multicolumn{1}{|c|}{ Fuente } & \multicolumn{1}{c|}{ Causa } \\
\hline Mano de Obra & $\begin{array}{l}\text { Al momento de almacenar las cajas de yogurt en las canastas y ubicar las mismas en } \\
\text { pilas se puede afectar el estado físico de las cajas debido a que las canastas son lanzadas } \\
\text { para su apilamiento. }\end{array}$ \\
- $\begin{array}{l}\text { Puede presentarse fallas en el producto terminado debido a que el operario no esperó el } \\
\text { tiempo suficiente para que la resistencia de la máquina se caliente lo suficiente y pueda } \\
\text { sellar la boca de la caja tetra pack de forma correcta. }\end{array}$ \\
- Incorrecta configuración de la máquina de envasado por parte del operario ocasionaría \\
un inadecuado contenido efectivo de producto (yogurt). \\
Al momento de trasladar la pila de canastas, las cajas de yogurt pueden sufrir rayas por \\
el gancho que utiliza el operario para jalar la pila.
\end{tabular}

Fuente: Adaptado de [4]. 
Tabla 2. Muestras del subproceso de envasado de yogurt pure pack.

\begin{tabular}{|c|c|c|c|}
\hline Subgrupo & $\begin{array}{c}\text { Defectos } \\
(\boldsymbol{D} \boldsymbol{i})\end{array}$ & Subgrupo & $\begin{array}{c}\text { Defectos } \\
(\boldsymbol{D} \boldsymbol{i})\end{array}$ \\
\hline 1 & 3 & 14 & 0 \\
\hline 2 & 0 & 15 & 0 \\
\hline 3 & 0 & 16 & 0 \\
\hline 4 & 3 & 17 & 2 \\
\hline 5 & 0 & 18 & 0 \\
\hline 6 & 0 & 19 & 0 \\
\hline 7 & 4 & 20 & 2 \\
\hline 8 & 2 & 21 & 4 \\
\hline 9 & 2 & 22 & 3 \\
\hline 10 & 3 & 23 & 0 \\
\hline 11 & 0 & 24 & 2 \\
\hline 12 & 1 & 25 & 0 \\
\hline 13 & 2 & & \\
\hline
\end{tabular}

Fuente: Los autores.

$$
\begin{gathered}
L S C=n \bar{p}+3 \sqrt{n \bar{p}(1-\bar{p})}=4,72 \\
L I C=n \bar{p}-3 \sqrt{n \bar{p}(1-\bar{p})}=0,0 \\
L C=n \bar{p}=1,32 \\
\bar{p}=\frac{\sum_{i=1}^{k} D i}{n \cdot k}=0,024
\end{gathered}
$$

Donde $D i$ es el número de cajas defectuosas por subgrupo, $n$ es el tamaño de la muestra (constante) y $k$ es el número de subgrupos. En este sentido, el tamaño de muestra $(n)$ en cada subgrupo fue de 54 cajas, suficientemente grande para garantizar una buena estimación de los parámetros. El valor de $n$ corresponde a cada lote de apilamiento de las cajas de yogurt (ver sección "VALIDACIÓN DEL DISEÑO METODOLÓGICO”). Se utilizó un $k=25$. El grafico de control establecido para el subproceso es presentado en la Figura 7.

\section{Análisis}

El análisis hecho en esta etapa toma en cuenta la clasificación de las no conformidades realizadas mediante el diagrama causa-efecto en Tabla 1, el análisis de la ocurrencia de los defectos a través del diagrama de Pareto en Figura 6 y el comportamiento del proceso representado por el gráfico de control estadístico $n p$ en Figura 7.

En los resultados se observa que aproximadamente el $87,34 \%$ de las no conformidades corresponden a dos defectos específicos: El "mal sellado de boca" y las "rayas en las cajas de yogurt purepack". Estos defectos son principalmente causados por las fuentes "Mano de obra" y "Máquinaría" (ver Tabla 1).

Por su parte, se observa como el gráfico de control $n p$ presenta el número de defectos en los 25 subgrupos analizados. En este sentido, se establece un límite superior de control LSC $=4,72$ con una media $n p=$ 1,32 cajas de yogurt no conformes. Note que ningún punto se encuentra fuera de los límites de control. Por lo tanto, no se puede rechazar la hipótesis de que el proceso se encuentra en estado de control estadístico con un nivel de confianza del $95 \%$.

Fuente: Los autores.

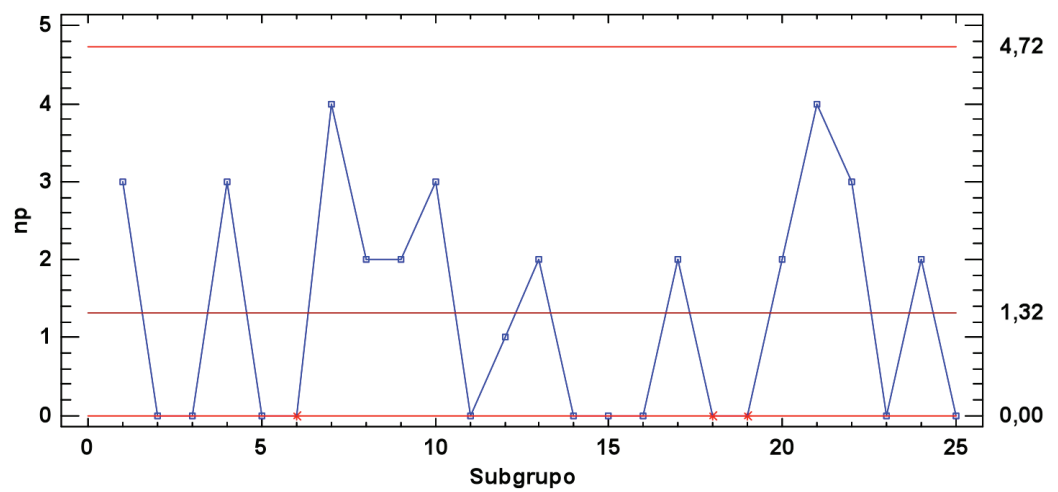

Figure 7. Gráfico de control establecido para el subproceso de envasado de yogurt purepack. 


\section{Mitigación}

En la etapa de mitigación se implementa el enfoque general del proceso de gestión de riesgos contenido en la norma ISO 31000 presentado en la sección de "INTRODUCCIÓN". Este proceso toma en consideración el tratamiento y gestión sobre las causas de no conformidades identificadas en fase de "identificación y descripción" del diseño metodológico mostrado en la sección "DISEÑO METODOLÓGICO”.

La gestión del riesgo implica que la empresa establezca elementos que sean adecuados en términos de infraestructura y cultura. Aplicando un método sistemático y lógico para establecer el contexto, identificando, evaluando, tratando, monitoreando y comunicando los riesgos asociados con cualquier actividad, función o proceso, de manera que permita a las organizaciones minimizar las pérdidas y maximizar los beneficios [5, 6, 23-25].

Establecimiento del contexto: El establecimiento de criterios de riesgo, cuyo impacto afectará la calidad del producto, los costos y los tiempos de producción, se determina por los procesos, etapas y actividades identificados. La Tabla 3 muestra los criterios y consecuencias para cada nivel de riesgo considerado en la presente investigación.

Identificación de riesgos: La identificación de los riesgos en términos de lo que puede suceder y cómo puede ocurrir, está contenida en la información recogida en las etapas de "Identificación y descripción" e "Implementación y clasificación" del diseño metodológico.

Análisis de riesgo: Se propone medir la posibilidad de ocurrencia del riesgo y sus consecuencias con base en la matriz de calificación y análisis de riesgo presentada en la Tabla 4. Las consecuencias determinan que tan grave es el riesgo para el proceso, mientras que la posibilidad es determinada por la frecuencia de la ocurrencia del mismo. La matriz de calificación y análisis de riesgo sugiere que cada riesgo sea evaluado tomando en cuenta los criterios establecidos en la matriz de criterios y consecuencias presentada en Tabla 3.

Evaluación del riesgo: La evaluación del riesgo se obtiene al multiplicar la posibilidad y las consecuencias de cada riesgo. La Tabla 4 muestra que, para cada nivel de consecuencia y posibilidad (en un rango de uno a cinco), el riesgo puede tomar valores de 1 a 25 , usándose como una medida de clasificación. En este contexto, los riesgos pueden ser clasificados como muy bajos, bajos, moderados, altos o muy altos. $\mathrm{La}$ información sobre la posibilidad y las consecuencias del riesgo fueron proporcionadas por personal directamente involucrado en el proceso de envasado.

Tratamiento de riesgos: En esta etapa, se implementa la "Matriz de acciones (MA)" representada por la

Tabla 3. Matriz de Criterios y Consecuencias.

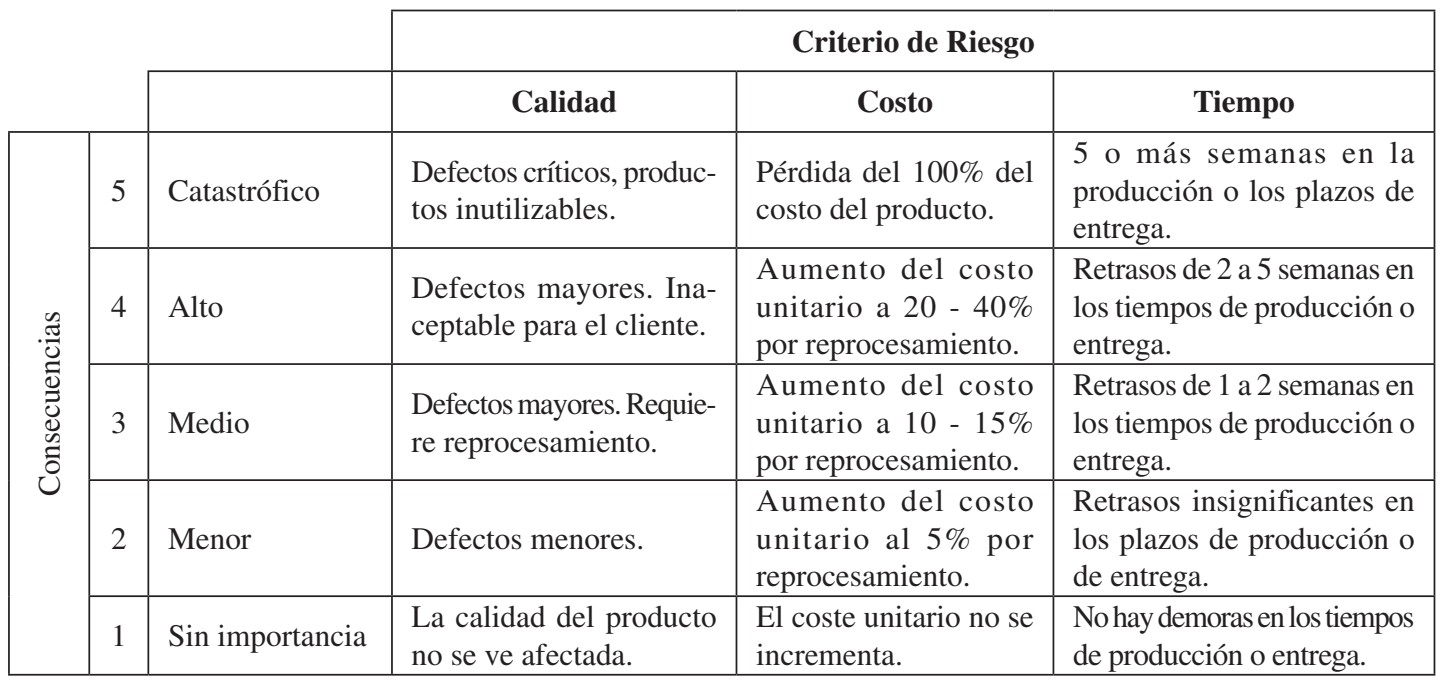

Fuente: Adaptado de [23-24]. 
Tabla 4. Matriz de calificación y análisis de riesgo.

\begin{tabular}{|c|c|c|c|c|c|c|}
\hline \multirow{7}{*}{ 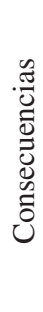 } & & 1 & 2 & 3 & 4 & 5 \\
\hline & & Excepcionalmente & Ocasionalmente & Regularmente & Generalmente & Siempre \\
\hline & 5 & 5 - Вајо & 10 - Moderado & 15 - Alto & 20 - Alto & 25 - Muy Alto \\
\hline & 4 & 4 - Bajo & 8 - Moderado & 12 - Alto & 16 - Alto & 20 - Alto \\
\hline & 3 & 3 - MuyBajo & 6 - Bajo & 9 - Moderado & 12 - Alto & 15 - Alto \\
\hline & 4 & 2 - Muy bajo & 4 - Вајо & 6 - Bajo & 8 - Moderado & 10 - Moderado \\
\hline & 1 & 1 - Muy bajo & 2 - Muy bajo & 3 - Muy Bajo & 4 - Bajo & 5 - Bajo \\
\hline
\end{tabular}

Fuente: Adaptado de [23-24].

Tabla 5, con el objetivo de proponer e implementar las acciones de contención, corrección y prevención necesarias para mitigar los riesgos de mayor prioridad. Como ejemplo, en la Tabla 5 muestra un resumen en la implementación de la matriz de acciones para el tratamiento de algunos de los riesgos identificados en el proceso de envasado de yogurt purepack.

La MA pretende identificar las acciones que minimicen el impacto de los riesgos o causas, que dan origen a las no conformidades del producto en las diferentes etapas productivas. Asimismo, recoge toda la información analizada y clasificada hasta esta etapa y, con base en el desarrollo de proceso de gestión de riesgos, propone un curso de acción para mitigar los riesgos inherentes al proceso y que son causa de las no conformidades en las cajas de yogurt purepack. A través de diversas acciones emprendidas sobre cada riesgo, se pretende reducir la posibilidad de ocurrencia y cualquier consecuencia negativa. La efectividad de estas acciones se reflejará en la reducción en la ocurrencia de riesgos y en la reducción de las no conformidades en el producto.

La estructura de la MA muestra secuencialmente las causas o riesgos identificados en las diferentes etapas del proceso. Se describen los riesgos y cómo se presentan. La evaluación del riesgo en términos de posibilidad y consecuencia también pueden ser encontradas. Esto con el fin de centrar la atención en los riesgos con más alta evaluación, ya que representan un mayor impacto en el deterioro de la calidad, el consumo de recursos y los retrasos en los tiempos de producción y entrega.

Adicionalmente, la MA presenta las acciones de contención, reacción y prevención en caso de ocurrencia del riesgo, permitiendo al operario del proceso tomar decisiones en tiempo real.

\section{Seguimiento y revisión}

Para el proceso de envasado de yogurt purepack se propone tomar muestras sobre la ocurrencia de los riesgos y actualizar las herramientas de análisis con una periodicidad no mayor a dos días. Esto, con el propósito de mantener el buen funcionamiento del diseño metodológico propuesto en la sección 2. Además, lo anterior ayudará a identificar cualquier nuevo riesgo que afecte el proceso o, por otro lado, reconocer si las acciones planteadas para mitigar los riesgos ya identificados han sido efectivas.

En esta investigación, se propone actualizar los riesgos de forma periódica teniendo en cuenta los recursos de la compañía. Al tratarse de un ciclo de mejora continua (Planear-Hacer-Verificar-Actuar) la organización debe corroborar que las acciones implementadas para reducir o eliminar los riesgos tengan un efecto positivo en la mejora del proceso. $\mathrm{Si}$ las acciones son eficaces y eliminan o reducen permanentemente algunos de los riesgos identificados, la gerencia debe mantener registros de los resultados que contribuyen al tema de las lecciones aprendidas dentro de la organización. En otro caso, se deben proponer nuevas acciones de contención, corrección y prevención.

\section{CONCLUSIONES}

Ha sido presentada una metodología de prevención de no conformidades en procesos de producción. La metodología pretende combinar herramientas estadísticas y de calidad con normas internacionales de gestión de riesgos. Convirtiéndose en una opción novedosa en la que es factible implementar un enfoque de riesgos en procesos productivos.

El diseño metodológico fue validado de manera satisfactoria permitiendo identificar, tratar, mitigar 
Tabla 5. Matriz de acciones para el proceso de envasado de yogurt purepack.

\begin{tabular}{|c|c|c|c|c|c|c|c|c|c|}
\hline 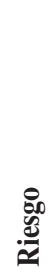 & 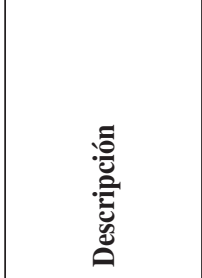 & 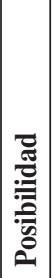 & 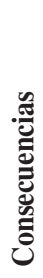 & 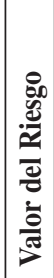 & 㺼 & 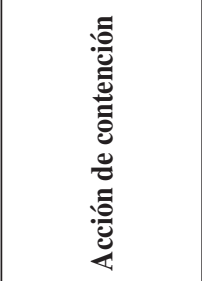 & 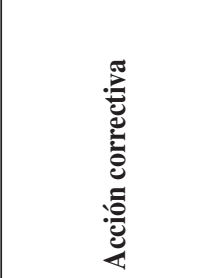 & 葛 & $\begin{array}{l}\frac{0}{0} \\
\frac{0}{0} \\
\overline{0} \\
\stackrel{0}{0} \\
\stackrel{0}{0}\end{array}$ \\
\hline 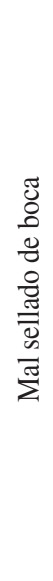 & $\begin{array}{l}\text { Puede presen- } \\
\text { tarse fallas en el } \\
\text { producto debido } \\
\text { a que el opera- } \\
\text { rio no esperó el } \\
\text { tiempo suficien- } \\
\text { te para que la } \\
\text { resistencia de } \\
\text { la máquina se } \\
\text { caliente lo su- } \\
\text { ficiente y pueda } \\
\text { sellar la boca } \\
\text { de la caja tetra } \\
\text { pack de forma } \\
\text { correcta. }\end{array}$ & 3 & 5 & 15 & Alto & $\begin{array}{l}\text { Retirar el pro- } \\
\text { ducto no confor- } \\
\text { me del área de } \\
\text { ubicación actual } \\
\text { determinando la } \\
\text { posibilidad de su } \\
\text { desecho definiti- } \\
\text { vo o reproceso. }\end{array}$ & $\begin{array}{l}\text { El operario debe } \\
\text { asegurarse y cer- } \\
\text { ciorarse de que la } \\
\text { resistencia de la } \\
\text { máquina logre su } \\
\text { máxima eficiencia, } \\
\text { para que esta haga } \\
\text { su función correc- } \\
\text { tamente. En caso } \\
\text { que la causa se dé } \\
\text { nuevamente se debe } \\
\text { llamar al personal } \\
\text { de mantenimiento. }\end{array}$ & $\begin{array}{l}\text { - Establecer y } \\
\text { seguir un inter- } \\
\text { valo de tiempo, } \\
\text { el cual permita } \\
\text { que la resistencia } \\
\text { de la máquina al- } \\
\text { cance su máxima } \\
\text { eficiencia. } \\
\text { - Garantizar la eje- } \\
\text { cución correcta } \\
\text { del programa de } \\
\text { mantenimiento } \\
\text { sobre la máquina } \\
\text { de fechado. }\end{array}$ & $\begin{array}{l}\text { Operario del } \\
\text { subproceso } \\
\text { - Supervisor }\end{array}$ \\
\hline 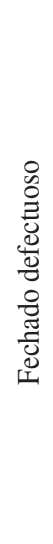 & $\begin{array}{l}\text { Se puede pre- } \\
\text { sentar poca } \\
\text { marcación de la } \\
\text { fecha de ven- } \\
\text { cimiento o del } \\
\text { lote sobre la } \\
\text { parte superior } \\
\text { del yogurt. }\end{array}$ & 2 & 5 & 10 & Moderado & $\begin{array}{l}\text { Retirar el pro- } \\
\text { ducto no confor- } \\
\text { me del área de } \\
\text { ubicación actual } \\
\text { determinando la } \\
\text { posibilidad de su } \\
\text { desecho definiti- } \\
\text { vo o reproceso. }\end{array}$ & $\begin{array}{l}\text { El operario debe } \\
\text { asegurarse y cer- } \\
\text { ciorarse de que la } \\
\text { resistencia de la } \\
\text { máquina logre su } \\
\text { máxima eficien- } \\
\text { cia, para que esta } \\
\text { haga un fechado } \\
\text { correcto. En caso } \\
\text { que la causa se dé } \\
\text { nuevamente se debe } \\
\text { llamar al personal } \\
\text { de mantenimiento. }\end{array}$ & $\begin{array}{l}\text { - Establecer y se- } \\
\text { guir un interva- } \\
\text { lo de tiempo, el } \\
\text { cual permita que } \\
\text { la resistencia de } \\
\text { la máquina al- } \\
\text { cance su máxima } \\
\text { eficiencia. } \\
\text { - Garantizar la eje- } \\
\text { cución correcta } \\
\text { del programa de } \\
\text { mantenimiento } \\
\text { sobre la máquina } \\
\text { de fechado. }\end{array}$ & $\begin{array}{l}\text { Operario del } \\
\text { subproceso } \\
\text { - Supervisor }\end{array}$ \\
\hline 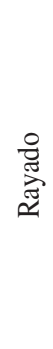 & $\begin{array}{l}\text { En ocasiones se } \\
\text { presentan rayas } \\
\text { en la parte su- } \\
\text { perior de la } \\
\text { caja de yogurt } \\
\text { ya envasada. }\end{array}$ & 2 & 3 & 6 & Bajo & $\begin{array}{l}\text { Retirar el pro- } \\
\text { ducto no confor- } \\
\text { me del área de } \\
\text { ubicación actual } \\
\text { determinando } \\
\text { la posibilidad } \\
\text { de su desecho } \\
\text { definitivo. }\end{array}$ & $\begin{array}{l}\text { El operario debe } \\
\text { garantizar la co- } \\
\text { rrecta utilización } \\
\text { del gancho que se } \\
\text { utiliza para jalar } \\
\text { la pila de cajas de } \\
\text { yogurt, evitando } \\
\text { el rayado de las } \\
\text { mismas. }\end{array}$ & $\begin{array}{l}\text { Se debe revisar nue- } \\
\text { vos métodos de api- } \\
\text { lamiento y traslado } \\
\text { de cajas, con el pro- } \\
\text { pósito de evitar el } \\
\text { uso de ganchos }\end{array}$ & $\begin{array}{l}\text { Gerencia - } \\
\text { Operador del } \\
\text { subproceso }\end{array}$ \\
\hline
\end{tabular}

Fuente: Adaptado de [22, 23, 24]. 
y monitorear las causas de desviaciones de especificaciones de producto en un proceso de envasado de yogurt purepack. Además, permitió identificar soluciones para mitigar causas o riesgos identificados como fuentes de no conformidades. Las herramientas estadísticas y de calidad ayudaron a procesar los datos recopilados, contribuyendo al análisis y al monitoreo del proceso. Del mismo modo, estas herramientas sirvieron para monitorear los cambios (positivos o negativos) causados por el tratamiento de los riesgos.

La implementación de la "Matriz de Acciones", basada en la ISO 31000 para la gestión de riesgos, nos permitió hacer un diagnóstico al proceso y proponer acciones para prevenir riesgos significativos para la organización. En este sentido, se implementaron herramientas que guían al operador del proceso de producción a tomar decisiones en tiempo real y prevenir o contener resultados indeseables. En futuras investigaciones se propone validar y comparar la metodología propuesta implementándola en distintos procesos de producción, incluyendo su adaptación a la producción de servicios.

\section{REFERENCIAS}

[1] J.C. García-Díaz y A. Pulido-Rojano. "D.C. Montgomery and control of the multihead weighing process through a modified control chart". DYNA. Vol. 84 N $^{\circ} 200$, pp. 135-142. 2017.

[2] A.Pulido-Rojano y C. Bocanegra-Bustamante. "Mitigación de defectos en productos manufacturados". Ingeniería y Competitividad. Vol. $17 \mathrm{~N}^{\circ}$ 1, pp. 161-172. 2015.

[3] A.J. Duncan. "Quality control and industrial statistics". Illinois: Irwin. 1986.

[4] D.C. Montgomery. "Introduction to Statistical Quality Control". 7th Edition. John Wiley \& Sons. New York, Estados Unidos. 2012.

[5] Australian/New Zealand Standard ISO 31000. "AS/NZ ISO 31000 Risk Management. Principles and Guidelines". Sidney: AS/NZ S Standards. 2009.

[6] NTC-5254 (Norma Técnica Colombiana). "Directrices para la Gestión de Riesgos". Bogotá, Colombia: ICONTEC. 2007.

[7] V. Svatá y M. Fleischmann. "Is/It Risk Management in Banking Industry". AOP. Vol. $19 \mathrm{~N}^{\circ}$ 3, pp. 42-60. 2011.
[8] J. Botín, R. Guzmán, y M. Smith. "A methodological model to assist in the optimization and risk management of mining investment decisions". DYNA. Vol. $78 \mathrm{~N}^{\circ} 170$, pp. 221-226. 2011.

[9] G. P. Gasca Hurtado. "Estudio de similitud del proceso de gestión de riesgos en proyectos de outsourcing de software: utilización de un método". Revista Ingenierías Universidad de Medellín. Vol. 9 No 17, pp. 119-130. 2010.

[10] C. López, and J.L. Salmerón. "Ranking risks in ambient intelligence projects". Journal of applied research and technology. $\mathrm{N}^{\circ} 9$, pp. 419-429. 2011.

[11] G. Martínez, B. Moreno y M.D. Rubio. "Gestión del riesgo en proyectos de ingeniería. El caso del campus universitario pts". Universidad de Granada (España). Journal Dyna. Vol. $79 \mathrm{~N}^{\circ}$ 173. 2012.

[12] A. Pérez-Vidal, C.P. Amézquita-Marroquín y P. Torres-Lozada. "Identificación y priorización de peligros como herramientas de la gestión del riesgo en sistemas de distribución de agua potable". Revista Ingeniería y Universidad. Vol. $16 \mathrm{~N}^{\circ}$ 2. 2012.

[13] R. Gómez, D. Pérez, Y. Donoso y A. Herrera. "Metodología y gobierno de la gestión de riesgos de tecnologías de la información". Revista de Ingeniería. № 31, pp. 109-118. 2010.

[14] C. Cachutt Alvarado, R.A. Illada García, J. R. Barreto Alvarado y F.E. Pinto Rodríguez. "Análisis de los procesos de fabricación en una empresa manufacturera mediante gráficos de control integrado". Revista Universidad, Ciencia y Tecnología. Vol. 12 $\mathrm{N}^{\circ}$ 49. 2008.

[15] E. Díaz, C. Díaz, L. Flores y S. Heyser. "Estudio de la Variabilidad de Proceso en el Área de Envasado de un Producto en Polvo". Revista Información tecnológica. Vol. $20 \mathrm{~N}^{\circ}$ 6, pp. 105-113. 2009.

[16] U. Pineda, M. Estrada y C.M. Parra. "Aplicación del modelo Servqual y herramientas de ingeniería de la calidad para la planificación del servicio en la Biblioteca Central de la Universidad de Antioquia". Revista Interamericana de Bibliotecología. Vol. 34 N $^{\circ}$ 3, pp. 243-255. 2011.

[17] P. Pérez. "Control estadístico de calidad multivariado, para el monitoreo e identificación de causas de variabilidad en procesos de 
crédito del sector financiero". 2012. Obtenido de Universidad Nacional: www.bdigital.unal. edu.co/8232/1/8910504.2012.pdf

[18] A. Zapata-Gómez. "Efecto de las técnicas de ingeniería de la calidad en el diseño de productos". Revista Ingeniería y Universidad. Vol. $17 \mathrm{~N}^{\circ}$ 2, pp. 409-425. 2013.

[19] L. Lee Ho y R. Costa Quinino. "An attribute control chart for monitoring the variability of a process". International Journal of Production Economics. Vol. $145 \mathrm{~N}^{\circ}$ 1, pp. 263-267. 2013.

[20] R. Peñabaena-Niebles, O. OviedoTrespalacios, S. Cuentas-Hernandez and E. García-Solano. "Methodology for the implementation of an economic and/or statistical design for $\mathrm{x}$-bar charts with variable parameters (VP)". DYNA. Vol. 81 $\mathrm{N}^{\circ} 184$, 2014. http://dx.doi.org/10.15446/ dyna.v81n184.35510.
[21] C. Berdugo-Correa, R. Barbosa-Correa y L. Prada-Angarita. "Variables relevantes para la medición de la calidad percibida del servicio bancario". DYNA. Vol. 83 No 197 , pp. 212-221. 2016.

[22] A. Pulido-Rojano. "Methodological design for the prevention of risk in production processes". DYNA. Vol. 82 No 193, pp. 16-22. 2015.

[23] P. Poveda Orjuela y M. Lopez Giraldo. "Configuración de herramientas guía para la gestión integral de riesgos de inocuidad según ISO 31000 e ISO 22000 (Vol. 1)”. Uninorte. Barranquilla, Colombia. 2010.

[24] P. Poveda Orjuela y G. Cañón Zabala. "Guía para la gestión integral de riesgos (Vol. 1)". ICONTEC, Bogotá, Colombia. 2015.

[25] NTC-ISO 31000 (Norma Técnica Colombiana). "Gestión del Riesgo. Principios y Directrices". ICONTEC, Bogotá, Colombia. 2011. 